
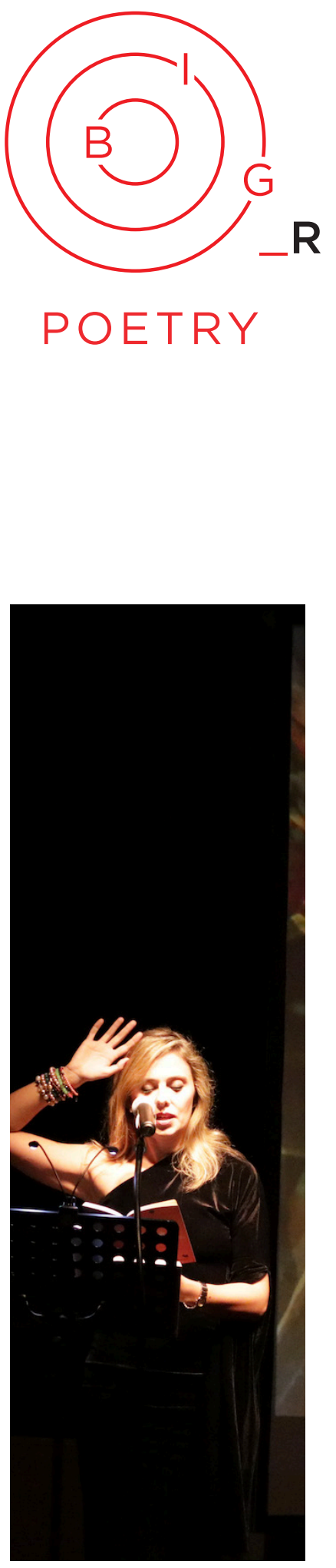

Borders in Globalization Review

Volume 1, Issue 1 (Fall 2019): 106-112 https://doi.org/10.18357/bigr11201919271

\title{
vagabond wind | sans-papiers
}

\author{
Natasha Sardzoska
}

\author{
Poems: \\ vagabond wind \\ sans-papiers \\ In English, Spanish, and French \\ Translations by Natasha Sardzoska (with Elsa Barreda Ruiz) \\ Originally published in: \\ Natasha Sardzoska, COCCYX (PNV Publishing, Skopje, 2019)
}

\section{About the Poet:}

Natasha Sardzoska (1979), poet, writer, essayist, polyglot translator and interpreter was born in Skopje in Macedonia. She holds a PhD in anthropology from the Eberhard Karls University of Tübingen, Sorbonne Nouvelle in Paris and University of Bergamo. She has published the poetry books Blue Room, Skin, He pulled me with invisible string, Living Water, Coccyx and short stories. Her book Skin was published in the USA and Italy; her poems are translated in international literary reviews. Her poem "Doll on Strings" has been published in the International Poetry Anthology against child abuse. She has performed at international poetry festivals among which: Ars Poetica in Bratislava; Words Wide Open in Genova; Scream in Rijeka; in the Revoltella Museum in Trieste; in the Academy of Arts for the Poetry Festival of Berlin; at the Sha'ar International Poetry Festival performing with sax, contrabass and contemporary dance in the Yaffa Theatre in Tel Aviv.

For more, see Natasha's Profile on Versopolis Poetry

Contact: sardzovskinatasha@gmail.com https://journals.uvic.ca/index.php/bigreview https://biglobalization.org/
Creative Commons

CC-BY-NC 4.0 


\title{
vagabond wind
}

\author{
silba el viento dentro de mí. \\ estoy desnudo. \\ dueño de nada, dueño de nadie, ni siquiera dueño de mis certezas, soy mi cara en el viento, a \\ contraviento, y soy el viento que me golpea en la cara. \\ eduardo galeano
}

\author{
at airports I am the traveler \\ examined for several times \\ random check they tell me \\ but I do not travel \\ nowhere \\ I tell them \\ I do not go anywhere \\ I do not even return \\ I am not a barbel in extinction \\ neither fardel which will determine the directions \\ they seek and scratch my bags \\ but I have nothing \\ nothing which will throw a shade on their fears \\ they ask me where do I go \\ but neither myself I do not know \\ which is the hotel address \\ what is written in the invitation letter \\ do I have a return ticket \\ I am a fish on dry soil \\ I tell them I want to move away \\ but I feel fear \\ can't you see \\ I do not have north nor south \\ I am the runway uprooted from your royal land \\ and still I am the absence of land \\ of your time \\ I am the hourglass \\ you cannot wait to trickle \\ nor to seep you \\ but you still seek \\ for the crumbling time \\ to your royal beginnings \\ to take you back
}


Borders in Globalization Review | Volume 1 | Issue 1 | Fall 2019

Sardzoska, "vagabond wind" and "sans-papiers"

\title{
sans-papiers
}

\author{
I am blowing the air around \\ I keep silent in their eyes \\ and they are running away \\ yes, they are probably running away \\ to relieve the turbines that I have drifted away \\ the lacuna carved by my spine \\ and they hit me with the pendulum of the space \\ they look at me with suspect and fear \\ they scratch me in their mind \\ and then they leave me \\ without seal without stamp \\ without humanity \\ and they send me beyond their borders \\ where I was a foreigner \\ but I did not remain \\ well I did not \\ even have a gamble to lose \\ to calm down my bones \\ and save them \\ from insanity
}




\section{viento vagabundo}

silba el viento dentro de mí. estoy desnudo. dueño de nada, dueño de nadie, ni siquiera dueño de mis certezas, soy mi cara en el viento, a contraviento, y soy el viento que me golpea en la cara.

eduardo galeano

en los aeropuertos soy el pasajero controlado muchas veces random check me dicen pero yo no viajo no voy a ninguna parte les digo ni me voy ni siquiera regreso no soy una especie en extinción ni el eje que determinará la dirección

ellos buscan en mis bolsas pero yo no tengo nada nada que opaque sus miedos me preguntan a dónde voy pero ni yo misma no lo sé ni cual es la dirección del hotel qué dice la carta del anfitrión y si tengo un boleto aéreo de regreso

soy un pescado en tierra seca

les digo que quiero escapar

pero tengo miedo

no ven

no tengo ni norte ni sur

una pista excavada en su tierra real

y sin embargo estoy ausente de la tierra

de su tiempo

soy el reloj de arena

a la que no esperan que caiga

ni que los deje

aunque estén buscando

una mancha de tiempo

que les devuelva

a su principio imperial 
Borders in Globalization Review | Volume 1 | Issue 1 | Fall 2019

Sardzoska, "vagabond wind" and "sans-papiers"

\section{sans-papiers}

estoy soplando el aire alrededor guardo mi silencio en sus ojos

y ellos están huyendo

probablemente están huyendo

para aliviar las turbinas que he hecho

las lagunas ahondadas de mi espina dorsal

y me pegan con el péndulo del espacio

me miran con duda y miedo

me rasgan en su miente

y luego me dejan sin sello ni timbre

sin humanidad

y me despachan detrás de las fronteras

donde era extranjero

aun no me quedé

y sin embargo

no tenía nada que perder

para calmar mis huesos

y para salvarlos

de la locura 


\section{le vent vagabond}

silba el viento dentro de mí. estoy desnudo. dueño de nada, dueño de nadie, ni siquiera dueño de mis certezas, soy mi cara en el viento, a contraviento, y soy el viento que me golpea en la cara.

eduardo galeano

sur les aéroports je suis le voyageur qui est inspecté plusieurs fois random check disent-ils mais je ne voyage

nulle part

je leur dis

je n'y vais pas

je ne rentre pas

je ne suis pas un barbeau en voie d'extinction

ni un fardeau qui déterminera la direction

ils fouillent mes sacs

et je n'ai

rien

rien qui puisse occulter leur peurs

ils me demandent où je vais

moi même ne le sais pas

et quelle est l'adresse de l'hôtel

et que dit la lettre de l'hôte

et si j'ai un billet d'avion aller-retour

je suis un poisson à terre sèche

je leur dis que je veux partir

mais j'ai peur

ne voyez pas

je n'ai ni nord ni sud

je suis une piste qui a été creusée sur votre terre royale

et pourtant je suis absence de terre

de votre temps

je suis le sablier

ne vous attendez pas à couler

ni à vous décoller

alors que vous êtes à la recherche du temps friable

qui à votre début impérial

vous remportera 
Borders in Globalization Review | Volume 1 | Issue 1 | Fall 2019

Sardzoska, "vagabond wind" and "sans-papiers"

\section{sans-papiers}

j'anéantis l'air autour de moi et je me tais dans leurs yeux et ils fuient

probablement ils fuient

pour soulager les turbines que j'ai fait bouger les lacunes creusées par ma colonne vertébrale

et ils me frappent avec le pendule de l'espace ils me regardent avec le doute et la peur ils me griffent dans leur cerveau et me laissent par la suite sans sceau sans timbres sans humanité

et ils m'envoyent derrière leurs frontières où j'étais un étranger mais je ne le suis pas resté alors que je n'avais même pas une garantie à perdre pour calmer mes os

et les sauver

de la folie 